\title{
Le blessé par attentat terroriste
}

\author{
Stéphane Mérat, Pierre Pasquier, Marie-Dominique Colas. Édition Arnette, 2017, 302 \\ pages. ISBN: 978-2-7-7184-1481-2
}

\author{
Daniel Audy, MD, FRCPC
}

Received: 6 January 2018/ Accepted: 11 January 2018/Published online: 19 January 2018

(C) Canadian Anesthesiologists' Society 2018

Ce livre se veut un outil de référence complet en matière de prise en charge des victimes d'attentats terroristes. Il propose la structure organisationnelle ainsi que les approches procédurales nécessaires à la gestion des victimes dès l'annonce de l'événement, et jusqu'à la fin des traitements requis. Soulignons la crédibilité incontestable de tous les auteurs sur le sujet. Il s'agit en effet, pour la plupart, de médecins militaires ou civils ayant œuvré sous l'égide de l'Organisation des Nations Unies ou de l'Organisation du Traité de l'Atlantique Nord lors de missions à l'étranger. Certains ont aussi été impliqués dans le traitement des blessés lors d'attentats terroristes commis en sol français.

L'écriture est fluide et les différents chapitres relèvent d'une taxonomie à la fois structurée et intuitive qui rend l'ouvrage facile à consulter. Les auteurs y proposent une approche globale, apte à orienter aussi bien les professionnels de la santé responsables d'initier la réponse à l'attentat que ceux qui auront pour mission d'en gérer les séquelles. Les principes et notions qui régissent le triage, lequel réfère à l'application de procédures ayant pour but de maximiser le nombre de survivants, y sont clairement expliqués. Peu de professionnels sont en effet rompus aux exigences imposées par des situations où l'ampleur de la demande dépasse les ressources en place. Le triage et ses principes sont alors nécessaires afin de guider une rationalisation nécessaire et qui vise à permettre la survie du plus grand nombre de victimes.

D. Audy, MD, FRCPC ( $)$

Département d'anesthésiologie, Université de Montréal,

Montréal, QC, Canada

e-mail: majordani17@yahoo.ca
L'ouvrage fait aussi une place de choix aux principes nécessaires au maintien de la sécurité du personnel soignant. Les attentats réunissent souvent des conditions dans lesquelles le respect des pratiques et des procédures les plus sécuritaires s'avère difficile à maintenir. La zone sinistrée est souvent source de menaces et le respect des règles a pour but d'éviter que les membres du personnel soignant ne grossissent les rangs des victimes, provoquant alors une réduction des services qui se double d'un accroissement de la charge imposée aux effectifs restants.

Les auteurs présentent de nombreuses statistiques émanant des conflits armés et des attentats perpétrés au cours des dernières années. On fait état des organes susceptibles d'être touchés et des principes thérapeutiques qui doivent régir les professionnels chargés d'intervenir. De nouveau, le pragmatisme d'un triage destiné à assurer la survie du plus grand nombre est mis en lumière. L'ouvrage contient plusieurs fiches, tableaux ou résumés qui facilitent la compréhension du lecteur tout en lui fournissant un éventail d'outils pratiques. Des sections du livre sont aussi consacrées aux armes de destruction massive, qu'elles soient de nature chimique, biologique ou nucléaire. Le livre se termine en soulignant l'importance du support psychologique dont doivent bénéficier les victimes, tant au moment de l'attentat que dans les mois et années qui suivront. Il souligne aussi l'importance qu'il faut accorder au bien-être psychologique du personnel soignant, lequel est parfois mis à rude épreuve.

Bref, cet ouvrage s'avère une référence incontournable pour tous les professionnels de la santé susceptibles d'être impliqués dans le soin des victimes d'actes terroristes. Il tire profit de la vaste expérience d'un personnel aguerri. Il nous rappelle que malgré le nombre restreint d'attentats et de victimes recensés en sol canadien, personne ne peut 
présumer être à l'abri des méfaits du terrorisme et de ses conséquences.
Responsabilité éditoriale Cet article a été traité par Dr Étienne de Médicis, rédacteur de la langue française, Journal canadien d'anesthésie.

\section{Conflit d'intérêt Aucun.}

